\title{
Fidelity Between Partial States as Signature of Quantum Phase Transitions
}

\author{
N. Paunković \\ SQIG - Instituto de Telecomunicações, IST, P-1049-001 Lisbon, Portugal \\ P. D. Sacramento, P. Nogueira and V. R. Vieira \\ CFIF and Department of Physics, IST, Technical University of Lisbon (TULisbon), P-1049-001 Lisbon, Portugal \\ V. K. Dugaev \\ Department of Mathematics and Applied Physics, Rseszów University of Technology, \\ Al. Powstańców Warszawy 6, 35-959 Rseszów, Poland
}

(Dated: November 5, 2018)

\begin{abstract}
We introduce a partial state fidelity approach to quantum phase transitions. We consider a superconducting lattice with a magnetic impurity inserted at its centre, and look at the fidelity between partial (either one-site or two-site) quantum states. In the vicinity of the point of the quantum phase transition, we observe a sudden drop of the fidelity between two one-site partial states corresponding to the impurity location and its close vicinity. In the case of two-site states, the fidelity reveals the transition point as long as one of the two electron sites is located at the impurity, while the other lies elsewhere in the lattice. We also determine the Uhlmann mixed state geometric phase, recently introduced in the study of the structural change of the system state eigenvectors in the vicinity of the lines of thermal phase transitions, and find it to be trivial, both for one- and two-site partial states, except when an electron site is at the impurity. This means that the system partial state eigenvectors do not contribute significantly to the enhanced state distinguishability around the point of this quantum phase transition. Finally, we use the fidelity to analyze the total amount of correlations contained within a composite system, showing that, even for the smallest two-site states, it features an abrupt quantitative change in the vicinity of the point of the quantum phase transition.
\end{abstract}

PACS numbers: 03.67.-a 05.70.Fh 75.40.Cx

Recently, considerable developments have been made within the research born out of the collaboration between physicists from two rather distinct fields, viz those of quantum information and computation and those of condensed matter and many-body physics. Most of this interdisciplinary work has focused on the realization of quantum information protocols with real many-body systems (for an overview, see [1]). Yet, a considerable development has been accomplished by moving in the "opposite" direction, ie applying concepts and techniques widely used within the field of quantum information and computation to the study of problems relevant within the field of condensed matter physics. One of such topics, recently researched to a great extent, is that of the use of entanglement in the study of zero-temperature quantum phase transitions [2], as well as general thermal phase transitions (for an overview, see [3], chapters 19-21, and references therein).

One application of the quantum information techniques to problems dealing with macroscopic many-body systems is the use of the (ground) state fidelity [4] in studying (quantum) phase transitions. It was first reported in [5], where the sudden drop of the fidelity along the regions of quantum phase transitions was observed in the Dicke and the $X Y$ models. Consequently, this method was applied to the cases of free Fermi systems [6], Bose-Hubbard models [7, 8], spin chains [9, 10] and in connecting the fidelity with the renormalization group flows [11]. Also, the cases of non-symmetry breaking quantum phase transitions that are not of the Landau-Ginzburg-Wilson type were studied [12, 13, 14]. A more formal and general study based on differential geometric insights, establishing a connection between quantum phase transitions and the relevant thermodynamic response functions, was developed in [15, 16] (see also [17]). Finally, using the mixed state fidelity, and extending the parameter space to include the temperature, quantum phase transitions were studied in [18], while the thermal phase transitions were explored in [15, 17, 19]. It is also worth noting here that the recently studied connection between the quantum phase transitions and Berry geometric phases [20] was established in [15, 16] on a more formal level, unifying the fidelity and the Berry phase approaches to quantum phase transitions. Subsequently, the Uhlmann mixed state geometric phase [21] (the generalization of the pure state Berry phase) was introduced in [19] as a tool in exploring the change of the system thermal equilibrium state eigenvectors in the vicinity of the line of a phase transition.

Given two physical systems $a$ and $b$, their quantum states, being either pure or mixed, are given by density operators $\hat{\rho}_{a}$ and $\hat{\rho}_{b}$. Since according to quantum mechanics it is in general not possible to unambiguously distinguish with probability one between two given states [1, 22], various measures of quantum state distinguishability have been 
introduced (for an overview, see [22]). One of the most widely used measures is (quantum) fidelity [4], given by the expression

$$
F\left(\hat{\rho}_{a}, \hat{\rho}_{b}\right)=\operatorname{Tr} \sqrt{\sqrt{\hat{\rho}_{a}} \hat{\rho}_{b} \sqrt{\hat{\rho}_{a}}}
$$

The numerical value of the fidelity ranges from $F=0$, in the case of fully distinguishable states [37], to $F=1$, when the two quantum states are completely indistinguishable from each other (ie they are identical). As it happened with most other quantum state distinguishability measures, the introduction of the quantum fidelity $F$ was also motivated by the analogous classical quantity. Classical fidelity $F_{c}$, a measure of distinguishability between two probability distributions $\left\{p_{a}(i)\right\}$ and $\left\{p_{b}(i)\right\}$, is defined as $F_{c}\left(p_{a}, p_{b}\right)=\sum_{i} \sqrt{p_{a}(i) p_{b}(i)}$. Measuring an observable $\hat{A}$ in states $\hat{\rho}_{a}$ and $\hat{\rho}_{b}$, one obtains probability distributions $\left\{p_{a}^{\hat{A}}(i)\right\}$ and $\left.\left\{p_{b}^{\hat{A}}(i)\right\}\right)$ respectively, whose mutual distinguishability is given by the classical fidelity $F_{c}\left(p_{a}^{\hat{A}}, p_{b}^{\hat{A}}\right)$. It can be proven (see [22]) that for any observable $\hat{A}$, the inequality $F\left(\hat{\rho}_{a}, \hat{\rho}_{b}\right) \leq F_{c}\left(p_{a}^{\hat{A}}, p_{b}^{\hat{A}}\right)$ holds, and there always exists an optimal observable $\hat{A}_{o p}$ for which the equality is achieved. In other words, quantum fidelity $F\left(\hat{\rho}_{a}, \hat{\rho}_{b}\right)$ gives the optimal value for the distinguishability between two quantum states, when compared through probability distributions $\left\{p_{a}^{\hat{A}}(i)\right\}$ ) and $\left\{p_{b}^{\hat{A}}(i)\right\}$ (note though that it does not give an optimal observable $\left.\hat{A}_{o p}\right)$. Using the modulus of an operator $\hat{R},|\hat{R}|=\left(\hat{R} \hat{R}^{\dagger}\right)^{1 / 2}$, one obtains an alternative expression for the fidelity [1]:

$$
F\left(\hat{\rho}_{a}, \hat{\rho}_{b}\right)=\operatorname{Tr}\left|\sqrt{\hat{\rho}_{a}} \sqrt{\hat{\rho}_{b}}\right|=\operatorname{Tr} \sqrt{\sqrt{\hat{\rho}_{a}} \sqrt{\hat{\rho}_{b}}\left(\sqrt{\hat{\rho}_{a}} \sqrt{\hat{\rho}_{b}}\right)^{\dagger}}=\operatorname{Tr} \sqrt{\sqrt{\hat{\rho}_{a}} \hat{\rho}_{b} \sqrt{\hat{\rho}_{a}}}
$$

The fidelity approach to quantum phase transitions is based on the use of the above quantity (1) in distinguishing macroscopic quantum states of many-body quantum systems. Consider a system given by the set of Hamiltonians $\hat{H}(q)$, where $q$ is a parameter representing a set of interaction coupling constants, which at $T=0$ is in a pure ground state $|\Phi\rangle=|\Phi(q)\rangle$. Then, the sudden drop of the fidelity (1) $F(|\Phi(q)\rangle\langle\Phi(q)|,| \Phi(\tilde{q})\rangle\langle\Phi(\tilde{q})|)=|\langle\Phi(q) \mid \Phi(\tilde{q})\rangle|$ between two ground states $|\Phi(q)\rangle$ and $|\Phi(\tilde{q})\rangle$ obtained for two close parameter points $q$ and $\tilde{q}=q+\delta q$, with $\delta q$ being small, can indicate the points of quantum phase transitions. The basic logic behind this idea is strikingly simple - two quantum states defining different macroscopic phases are expected to have enhanced distinguishability that would quantitatively exceed that taken between the states from the same phase. In our study, instead of the global ground states that describe the overall system, we will study partial quantum states of certain subsystems of the system. If the overall system $\mathcal{S}$ is divided into two parts $A$ and $B$, then we will study the mixed state fidelity $F\left(\hat{\rho}_{A}(q), \hat{\rho}_{A}(\tilde{q})\right)$, where $\hat{\rho}_{A}(q)=\operatorname{Tr}_{B}|\Phi(q)\rangle\langle\Phi(q)|$, and analogously for $\hat{\rho}_{A}(\tilde{q})$. We will show that in the system considered, a conventional BCS superconductor with an inserted magnetic impurity, a sudden drop of the above mixed state fidelity can define the point at which the (first order) quantum phase transition occurs, even when applied to the smallest subsystems of the overall system.

\section{THE MODEL}

In this work we consider the quantum phase transition induced by a magnetic impurity inserted in a conventional superconductor [23, 24, 25, 26, 27, 28, 29, 30]. Consider a classical spin immersed in a two-dimensional $s$-wave conventional superconductor. We use a lattice description of the system. In the centre of the system, $i=l_{c}=\left(x_{c}, y_{c}\right)$, we place a classical spin parametrized by

$$
\frac{\vec{S}}{S}=\cos \varphi \vec{e}_{x}+\sin \varphi \vec{e}_{z}
$$

The Hamiltonian of the system is given by:

$$
\hat{H}=-\sum_{\langle i j\rangle \sigma} t_{i j} \hat{c}_{i \sigma}^{\dagger} \hat{c}_{j \sigma}-\varepsilon_{F} \sum_{i \sigma} \hat{c}_{i \sigma}^{\dagger} \hat{c}_{i \sigma}+\sum_{i}\left(\Delta_{i} \hat{c}_{i \uparrow}^{\dagger} \hat{c}_{i \downarrow}^{\dagger}+\Delta_{i}^{*} \hat{c}_{i \downarrow} \hat{c}_{i \uparrow}\right)-\sum_{\sigma \sigma^{\prime}} J\left[\cos \varphi \hat{c}_{l_{c} \sigma}^{\dagger} \sigma_{\sigma \sigma^{\prime}}^{x} \hat{c}_{l_{c} \sigma^{\prime}}+\sin \varphi \hat{c}_{l_{c} \sigma}^{\dagger} \sigma_{\sigma \sigma^{\prime}}^{z} \hat{c}_{l_{c} \sigma^{\prime}}\right] .
$$

where the first term describes the hopping of electrons between different sites on the lattice, $\varepsilon_{F}$ is the chemical potential (equal to the Fermi energy at $T=0$ ), the third term is the superconducting $s$-pairing with the site-dependent order parameter $\Delta_{i}$, and the last term with $J>0$ is the exchange interaction of an electron at site $i=l_{c}$ with the magnetic impurity. The hopping matrix is given by $t_{i j}=t \delta_{j, i+\delta}$ where $\delta$ is a vector to a nearest-neighbor site. Note that both 
the indices $l$ and $i, j \in\{1,2, \ldots N\}$ specify sites on a two-dimensional system ( $N$ is the number of sites). We will take energy units in terms of $t(t=1)$, and $\varepsilon_{F}=-1$.

Since the impurity spin acts like a local magnetic field the electronic spin density will align along the local spin. For small values of the coupling there is a negative spin density around the impurity site. At the impurity site it is positive, as expected. For larger couplings the spin density in the vicinity of the impurity site is positive. At small couplings the many-body system screens the effect induced by the impurity inducing fluctuations that compensate the effect of the local field in a way that the overall magnetization vanishes. However, for a strong enough coupling the many-body system becomes magnetized in a discontinuous fashion. One interpretation is that if $J$ is strong enough the impurity breaks a Cooper pair and captures one of the electrons, leaving the other electron unpaired, and thus the overall electronic system becomes polarized. The impurity induces a pair of bound states inside the superconducting energy gap, one at positive energy (with respect to the chemical potential), and another at a symmetric negative energy. Even though the spectrum is symmetric the spectral weights of the two energy levels are not the same and their spin content is also distinct. The analysis of the local density of states (LDOS) [27, 31] shows that for small coupling the lowest positive energy level has only a contribution from spin $\uparrow$ and the first level with negative energy (symmetric to the other level) has only a contribution from spin $\downarrow$. The magnitude of the spectral weight at the impurity site is different for the two states. Considering a higher value for the coupling one finds that the levels inside the gap approach the Fermi level. There is a critical value of the coupling for which the two levels cross in a discontinuous way such that it coincides with the emergence of a finite overall magnetization. After the level crossing occurs, the nature of the states changes. The positive energy bound state has now only a contribution from the spin $\downarrow$ component and vice-versa, the first negative energy state has only contribution from the spin component $\uparrow$ - hence as the level crossing takes place the spin content changes.

The diagonalization of this Hamiltonian is performed using the Bogoliubov-Valatin transformation in the form:

$$
\begin{aligned}
& \hat{c}_{i \uparrow}=\sum_{n}\left[u_{n}(i, \uparrow) \hat{\gamma}_{n}-v_{n}^{*}(i, \uparrow) \hat{\gamma}_{n}^{\dagger}\right] \\
& \hat{c}_{i \downarrow}=\sum_{n}\left[u_{n}(i, \downarrow) \hat{\gamma}_{n}+v_{n}^{*}(i, \downarrow) \hat{\gamma}_{n}^{\dagger}\right] .
\end{aligned}
$$

Here $n$ is a complete set of states, $u_{n}$ and $v_{n}$ are related to the eigenfunctions of the Hamiltonian (4), and the new fermionic operators $\hat{\gamma}_{n}$ are the quasiparticle operators. These are chosen such that in terms of the new operators:

$$
\hat{H}=E_{g}+\sum_{n} \epsilon_{n} \hat{\gamma}_{n}^{\dagger} \hat{\gamma}_{n},
$$

where $E_{g}$ is the ground state energy and $\epsilon_{n}$ are the excitation energies. As a consequence:

$$
\begin{aligned}
& {\left[\hat{H}, \hat{\gamma}_{n}\right]=-\epsilon_{n} \hat{\gamma}_{n}} \\
& {\left[\hat{H}, \hat{\gamma}_{n}^{\dagger}\right]=\epsilon_{n} \hat{\gamma}_{n}^{\dagger} .}
\end{aligned}
$$

The coefficients $u_{n}(i, \sigma), v_{n}(i, \sigma)$ can be obtained solving the Bogoliubov - de Gennes (BdG) equations [32]. Defining the vector:

$$
\psi_{n}(i)=\left(\begin{array}{c}
u_{n}(i, \uparrow) \\
v_{n}(i, \downarrow) \\
u_{n}(i, \downarrow) \\
v_{n}(i, \uparrow)
\end{array}\right)
$$

the $\mathrm{BdG}$ equations can be written as:

$$
\mathcal{H} \psi_{n}(i)=\epsilon_{n} \psi_{n}(i),
$$

where the $\mathcal{H}$ matrix at site $i$ is given by:

$$
\mathcal{H}=\left(\begin{array}{cccc}
-h-\varepsilon_{F}-J \sin \varphi & \Delta_{i} & -J \cos \varphi & 0 \\
\Delta_{i}^{*} & h+\varepsilon_{F}-J \sin \varphi & 0 & -J \cos \varphi \\
-J \cos \varphi & 0 & -h-\varepsilon_{F}+J \sin \varphi & \Delta_{i} \\
0 & -J \cos \varphi & \Delta_{i}^{*} & h+\varepsilon_{F}+J \sin \varphi
\end{array}\right),
$$


where $h=t \hat{s}_{\delta}$ with $\hat{s}_{\delta} f(i)=f(i+\delta)$. The solution of these equations gives both energy eigenvalues and eigenstates. The problem involves the diagonalization of a $(4 N) \times(4 N)$ matrix. The solution of the BdG equations is performed self-consistently imposing at each iteration that

$$
\Delta_{i}=\frac{g}{2}\left[\left\langle\hat{c}_{i \uparrow} \hat{c}_{i \downarrow}\right\rangle-\left\langle\hat{c}_{i \downarrow} \hat{c}_{i \uparrow}\right\rangle\right]
$$

where $g$ is the effective attractive interaction between the electrons. Using the canonical transformation this can be written as

$$
\Delta_{i}=-g \sum_{n, 0<\epsilon_{n}<\hbar \omega_{D}}\left\{f_{n}\left(\epsilon_{n}\right)\left[u_{n}(i, \uparrow) v_{n}^{*}(i, \downarrow)+u_{n}(i, \downarrow) v_{n}^{*}(i, \uparrow)\right]-\frac{1}{2}\left[u_{n}(i, \uparrow) v_{n}^{*}(i, \downarrow)+u_{n}(i, \downarrow) v_{n}^{*}(i, \uparrow)\right]\right\}
$$

where $\omega_{D}$ is the Debye frequency, and $f_{n}\left(\epsilon_{n}\right)$ is the Fermi function defined as usual like

$$
f_{n}\left(\epsilon_{n}\right)=\frac{1}{e^{\epsilon_{n} / T}+1}
$$

where $T$ is the temperature. We note that the Bogoliubov - de Gennes equations are invariant under the substitutions $\epsilon_{n} \rightarrow-\epsilon_{n}, u(\uparrow) \rightarrow v(\uparrow), v(\uparrow) \rightarrow u(\uparrow), v(\downarrow) \rightarrow-u(\downarrow), u(\downarrow) \rightarrow-v(\downarrow)$.

\section{RESULTS}

As we have already noted, in this paper we focus on the fidelity between the partial states rather than the overall ground states, as it was the case in the previous studies [5, 6, 7, 9 , 11, 12, 13, 14, 15, 16, 17, 18, 19]. At $T=0$, the system is in the pure ground state $|\Phi\rangle$ and has a density operator $\hat{\rho}=|\Phi\rangle\langle\Phi|$. Thus, if we divide the whole system in two subsystems, say $A$ and $B$, then the partial mixed state, given by the reduced density operator $\hat{\rho}_{A}$ for the subsystem $A$, is defined as

$$
\hat{\rho}_{A}=\operatorname{Tr}_{B} \hat{\rho},
$$

where $\operatorname{Tr}_{B}[\cdot]$ represents the partial trace evaluated over the Hilbert space $\mathcal{H}_{B}$ of the subsystem $B$. In particular, we will calculate the matrix elements of one-site and two-site partial mixed states, given in general for different sites $i$ and $(i, j)$ respectively, and different values of the coupling constant $J$. In order to infer the point of the quantum phase transition, we will calculate the fidelity between two one-site and two two-site states, given for two close parameters $J$ and $J+\delta J$. Further, to better understand the influence of the quantum phase transition point on the structure of partial states (and therefore, to a certain extent, the overall state as well), we examine the fidelity between two different partial states, given for the same values of the coupling constant $J$, but different sites, for both cases of oneand two-site states.

In many-body systems a description in terms of quantum pure states and wave functions as their representatives in a certain basis (e.g. position, momentum, energy,...) is quite involved and the second quantization is the natural way to perform any calculation. The mixed states are however easily defined in terms of pure states and their matrix elements in some basis, and this approach is indeed not difficult to implement using Fock states. The matrix elements of the density matrix are simply defined in terms of correlation functions of the whole system. For instance, in the case of the single-site partial mixed states it can be shown that, using local basis states $\mathcal{B}=\{|0\rangle,|\uparrow \downarrow\rangle|\uparrow\rangle,|\downarrow\rangle\}$, which denote the four possible states - unoccupied, double occupied, single occupied with an electron with spin up and single occupied with an electron with spin down, respectively — the corresponding density matrix reads as

$$
\rho_{i}=\left(\begin{array}{cccc}
\left\langle\left(1-\hat{n}_{\uparrow}\right)\left(1-\hat{n}_{\downarrow}\right)\right\rangle & \left\langle\hat{c}_{\uparrow}^{\dagger} \hat{c}_{\downarrow}^{\dagger}\right\rangle & 0 & 0 \\
\left\langle\hat{c}_{\downarrow} \hat{c}_{\uparrow}\right\rangle & \left\langle\hat{n}_{\uparrow} \hat{n}_{\downarrow}\right\rangle & 0 & 0 \\
0 & 0 & \left\langle\hat{n}_{\uparrow}\left(1-\hat{n}_{\downarrow}\right)\right\rangle & \left\langle\hat{c}_{\downarrow}^{\dagger} \hat{c}_{\uparrow}\right\rangle \\
0 & 0 & \left\langle\hat{c}_{\uparrow}^{\dagger} \hat{c}_{\downarrow}\right\rangle & \left\langle\left(1-\hat{n}_{\uparrow}\right) \hat{n}_{\downarrow}\right\rangle
\end{array}\right)_{i}
$$

where the index $i$ denotes the site. The spin and the charge parts decouple. The spin part couples the two spin orientations (single occupied states) and the charge part couples the empty and doubly occupied states. The diagonal terms of the matrix describe the number of empty sites, the number of doubly occupied sites, the number of spin up sites and the number of spin down sites, respectively. The sum of the diagonal terms is equal to 1 due to normalization. The matrix is easily diagonalized and the fidelity (1) between two different one-site states obtained straightforwardly. 


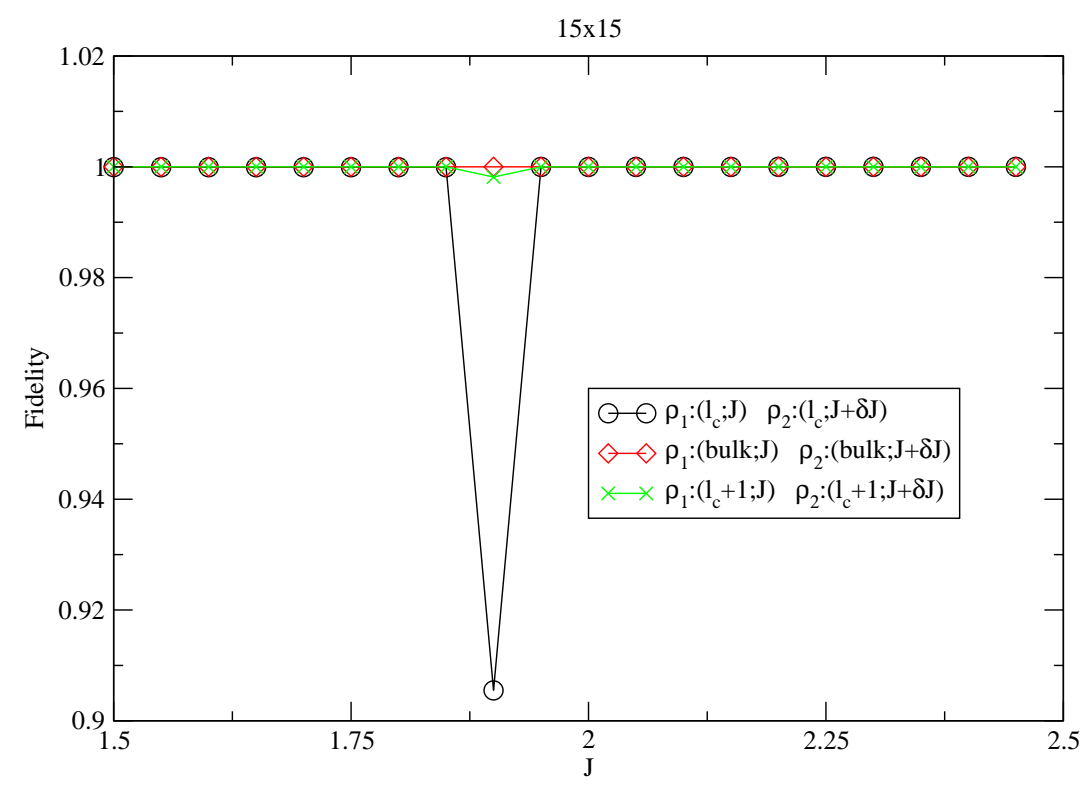

FIG. 1: One-site fidelity between two states taken for two close parameter points $J$ and $J+\delta J(\delta J=0.05)$, for the site at the impurity (black line), a nearest neighbor (green line) and a site located in the bulk (red line). Note the sudden drop of the fidelity for the impurity around the point of the quantum phase transition.

The correlation functions are easily solved using the representation of the electron operators in terms of the BdG quasiparticle operators. Specifically, we can write the single-site density matrix as

$$
\hat{\rho}=\sum_{n, m} \rho_{n m}|n\rangle\langle m|
$$

where $|n\rangle,|m\rangle \in \mathcal{B}$ are the four basis states described above. Consider now an operator $\hat{O}$ defined in terms of creation and annihilation operators of the electrons, and evaluate

$$
\operatorname{Tr}\{\hat{O} \hat{\rho}\}=\sum_{n, m}\langle n|\hat{O}| m\rangle \rho_{m n}
$$

To determine the matrix element $\rho_{n m}$ of the density matrix it is enough, by inspection, to find which operator $\hat{O}$ is such that only the matrix element $\langle n|\hat{O}| m\rangle$ is nonzero. This will tell us which correlation function gives which matrix element of the density matrix. Alternatively, one can express the operator $|m\rangle\langle n|$ in terms of the creation and annihilation operators. The results for $\rho_{A}$ are given by equation (15) above.

These matrix elements are defined in the subspace of the states of one site. However, since we have integrated out all the other sites, we may now replace the matrix elements by the matrix elements over the entire system (direct product with the states of the remaining $N-1$ sites).

Computing the fidelity between the two-site partial states is more involved. Given two sites, $i$ and $j$, the two-site basis states are given by the direct product of the one-site basis sets that were defined earlier, and the reduced density matrix is now a square matrix of order 16 . However, exactly half of the 256 matrix elements are null - the nonzero ones being those that correspond to either even-even or odd-odd states, where the parity reflects the number of electrons in the sites $i$ and $j$, respectively. Hence those two sets of states decouple and the reduced density matrix can be written as a block diagonal matrix where each submatrix is a square matrix or order 8 . Still, the calculation is rather complex and for that reason we used the computer program FORM [33] to obtain symbolic expressions for the matrix elements involved. Note that one of the correlation functions involves the double occupancies at the two sites, and thus one should deal with products of up to eight electron operators (where each electron operator is given by a sum of two quasiparticle operators); also, the signs that follow from the anti-commutation relations of fermionic operators must be taken into account. 

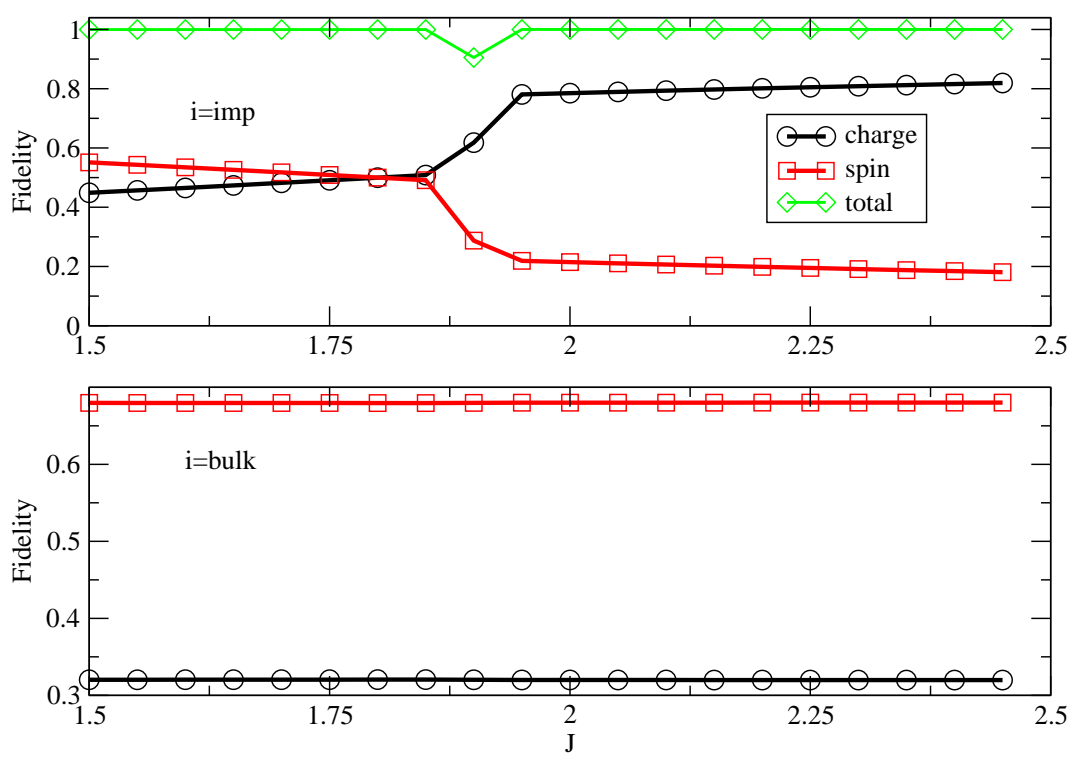

FIG. 2: The charge and spin contributions of the one-site fidelity as a function of the parameter $J$, with $\delta J=0.05$, for the site at the impurity (upper figure) and a site located in the bulk (lower figure). Note the discontinuity for both spin and charge contributions around the point of the quantum phase transition, for the site located at the impurity.

\section{Fidelity Between Single-site States}

In the following, we present the numerical results for the fidelity between one-site partial states, for a two dimensional lattice of size $15 \times 15$ sites. For computational simplicity, we perform our calculations for this system size. Since the effect of the impurity is short-ranged, changing the size of the system only affects slightly the results. First, we evaluate the fidelity (11) between two one-site states given for two sites $i$ and $j$ and two close values $J$ and $J+\delta J$ of the exchange parameter, with $\delta J=0.05$, such that $\hat{\rho}_{a}=\hat{\rho}(J ; i)$ and $\hat{\rho}_{b}=\hat{\rho}(J+\delta J ; j)$.

For $i=j=l_{c}$, when the electrons are located at the site of the magnetic impurity, the results for the one-site fidelity $F_{1}\left(\hat{\rho}_{a}, \hat{\rho}_{b}\right)$, as a function of $J$, are presented in Fig. 1. We observe a clear drop, from the otherwise common value $F_{1}\left(\hat{\rho}_{a}, \hat{\rho}_{b}\right) \simeq 1$, for the critical value $J_{0} \simeq 1.9$ [31] of the parameter $J$. As we move one of the electrons from the impurity, the drop of the fidelity $F_{1}$ around the point of the quantum phase transition becomes much smaller: for the first neighbors of the impurity, it is still visible, while already for the second-neighbors and the far away bulk sites, it becomes negligible. We see that even for the smallest subsystems - one-site spins in the lattice - the fidelity can be dramatically influenced by the structure of their states, as long as they are close to the impurity. This is intuitively easy to accept since it was shown in the previous work [31, 34], through investigation of various relevant physical quantities, such as the gap, the electron density or the total and quantum correlations (entanglement), that the impurity affects one-site properties of the system mainly in its vicinity (although it was also shown that for some two-site entanglement measures the effects persisted for larger distances).

A more detailed picture of the fidelity between the one-site states is presented in Fig. 2 where the two distinct contributions to the overall change of partial states is presented. The charge and the spin parts of the one-particle state (15) lead to two different contributions to the state distinguishability, and we see that they both exhibit a discontinuity in the vicinity of the point of the quantum phase transition.

Further, we have evaluated the Uhlmann mixed state geometric phase [21], a generalization of the Berry geometric phase to mixed quantum states. While the connection between Berry phases, pure state fidelity and quantum phase transitions was studied before [15, 16, 20], the Uhlmann mixed state geometric phase was recently introduced [19] to analyze the structural change of the system eigenvectors, with respect to the change of the relevant parameter driving the system Hamiltonian (ie $J$ in our case). The Uhlmann geometric phase $\phi_{g}$ (and the corresponding holonomy) is determined by the unitary operator $\hat{V}_{a b}$, given by the polar decomposition (see for example [1]) $\sqrt{\hat{\rho}_{a}} \sqrt{\hat{\rho}_{b}}=\left|\sqrt{\hat{\rho}_{a}} \sqrt{\hat{\rho}_{b}}\right| \hat{V}_{a b}$, where $|\hat{R}|=\left(\hat{R} \hat{R}^{\dagger}\right)^{1 / 2}$ represents the modulus of the operator $\hat{R}$ (for details, see [35]). Since the fidelity can be written as $F\left(\hat{\rho}_{a}, \hat{\rho}_{b}\right)=\operatorname{Tr}\left|\sqrt{\hat{\rho}_{a}} \sqrt{\hat{\rho}_{b}}\right|$, see equation (2) , a nonzero difference $H\left(\hat{\rho}_{a}, \hat{\rho}_{b}\right)-F\left(\hat{\rho}_{a}, \hat{\rho}_{b}\right)$, where $H\left(\hat{\rho}_{a}, \hat{\rho}_{b}\right)=\operatorname{Tr}\left[\sqrt{\hat{\rho}_{a}} \sqrt{\hat{\rho}_{b}}\right]$, would imply $\hat{V}_{a b} \neq \hat{I}_{a b}$ and therefore the emergence of a non-trivial Uhlmann geometric phase $\phi_{g} \neq 0$. On the other hand, for the case of mutually commuting Hamiltonians the system eigenvectors do 
not change with the driving parameter and we have $F\left(\hat{\rho}_{a}, \hat{\rho}_{b}\right)=\operatorname{Tr}\left[\sqrt{\hat{\rho}_{a}} \sqrt{\hat{\rho}_{b}}\right]$. In other words, $F\left(\hat{\rho}_{a}, \hat{\rho}_{b}\right)=H\left(\hat{\rho}_{a}, \hat{\rho}_{b}\right)$ and the Uhlmann geometric phase becomes trivial, ie $\phi_{g}=0$.

For the one-site states, we find that the Uhlmann geometric phase is always trivial for every site neighboring the impurity or in the bulk: the one-site eigenvectors do not contribute significantly to the change of the overall one-site partial states at the point of the quantum phase transition. Still, for the impurity site itself, around the point of the phase transition $J_{0}$ we observe a small deviation from zero for the difference $H_{1}-F_{1}$, which indicates a slight "rotation" of the electron's eigenbasis. This result can be qualitatively explained noticing that in the model we are considering the third term in the Hamiltonian (4), giving the superconducting features, and the last term in the Hamiltonian, giving the coupling between the impurity and the lattice electrons, commute with each other [38]. Moreover, for a fixed value of the gap $\Delta_{i}$, changing the impurity-lattice coupling constant $J$, without changing the impurity spin orientation angle $\varphi$, will result in no change of the overall Hamiltonian's eigenbasis — only the eigenvalues are affected in this case and the system exhibits a first-order quantum phase transition as a consequence of the explicit (not avoided) level crossing. Thus, one might expect that also the partial subsystem states would feature little change of local eigenbasis, even in the vicinity of the point of the quantum phase transition. This is precisely the case in the model considered, apart for the gap $\Delta_{l_{c}}$ given for the electron located at the impurity (see [31]): for every site $i$, the gap $\Delta_{i}$ is a slowly changing function of $J$, while for the impurity $\Delta_{l_{c}}$ exhibits a $\pi$ shift and becomes negative. Such a behavior of $\Delta_{l_{c}}$ does affect the difference $H_{1}-F_{1}$, but the effect is an order of magnitude smaller than for the fidelity. Further, it affects the impurity site only and thus does not lead to the superconductor-normal metal type of phase transition.

Finally, to further investigate the structure of one-site partial states, we have evaluated the fidelity between the states for two different sites, and the same value of the parameter $J$. The results are presented in Fig. 3. Again, we see that apart from the state of the site $l_{c}$ located at the impurity, and those at its vicinity (first neighbors), all the other one-site partial bulk states are almost the same, for a given common value of the parameter $J$. In Fig. 3 we have presented the results for two distinct values of the parameter, $J^{-}=1.5<J_{0}$ and $J^{+}=2.5>J_{0}$, but the qualitative picture stays the same for every $J^{-}<J_{0}$ and $J^{+}>J_{0}$ : the numerical value for the fidelity between the bulk state (say, $\hat{\rho}_{1}$ ) and the impurity state (say, $\hat{\rho}_{2}$ ) is significantly greater for $J^{-}$than for $J^{+}$, in other words, $F_{1}^{-}\left(\hat{\rho}_{a}, \hat{\rho}_{b}\right)>F_{1}^{+}\left(\hat{\rho}_{a}, \hat{\rho}_{b}\right)$, thus revealing the point of the quantum phase transition.

Note that when $i=l_{c}$ and $J<J_{0}$, besides the peak at the impurity site, in its vicinity the fidelity $F_{1}$ is smaller than the background value, while for $J>J_{0}$ it is larger. This is directly connected to the existence of oscillations of the magnetization around the impurity site (see 31]). Within the bounds set by quantum mechanics, two quantum states can be distinguished by measuring different observables and comparing the outcomes obtained. Magnetization is just one of them, and in this case we see that it is optimal, or at least nearly so: for $J<J_{0}$, it is easier to distinguish the impurity site from a site in its immediate vicinity than from other, more distant sites, simply because the difference in the expected magnetization between two sites is bigger in the case of impurity-neighborhood than in other cases. Therefore, in this case the fidelity has a drop in the neighborhood of the impurity, ie the distinguishability is larger there than elsewhere. A similar analysis holds for $J>J_{0}$.

\section{Fidelity Between Two-site States}

We have also studied the behavior of the fidelity taken between two-site partial states. We considered two distinct cases. First, we calculated the fidelity between two-site states for which one of the sites $i, j$ is at the impurity, say $i=l_{c}$, and the other is in its vicinity, $j=l_{c}+\delta l(\delta l=1,2, \ldots 6)$, for two close parameter values $J$ and $J+\delta J$, with $\delta J=0.05$. In Fig. 4 we present the results for the two-site fidelity $F_{2}\left(\hat{\rho}_{a}, \hat{\rho}_{b}\right)$, with $\hat{\rho}_{a}=\hat{\rho}\left(J ; l_{c}, l_{c}+\delta l\right)$ and $\hat{\rho}_{b}=\hat{\rho}\left(J+\delta J ; l_{c}, l_{c}+\delta l\right)$, where for clarity we plotted only the $\delta l=1,2,3$ cases, as the other curves almost overlap the results for $\delta l=3$. Again, as in the previous case of the fidelity between the one-site states, here as well the sudden drop of the fidelity from its common value $F_{2}\left(\hat{\rho}_{a}, \hat{\rho}_{b}\right) \simeq 1$ can be observed precisely in the vicinity of the critical point $J_{0}=1.9$. We see it as a signature of both the change in the impurity one-site partial states, as well as the change of the total correlations between the impurity and the remaining sites. From our study of the one-site fidelity, we have seen that the bulk partial states are all approximately the same, irrespectively of the site and the parameter value, while only the impurity and first neighbor one-site states exhibit a visible dramatic change around the point of the quantum phase transition $J_{0}$.

On the other hand, for every value of $J$ we can consider the quantity

$$
C_{2}\left(J, J+\delta J ; l_{c}, l_{c}+\delta l\right) \equiv \frac{F_{2}\left(\hat{\rho}\left(J ; l_{c}, l_{c}+\delta l\right), \hat{\rho}\left(J+\delta J ; l_{c}, l_{c}+\delta l\right)\right)}{F_{1}\left(\hat{\rho}\left(J ; l_{c}\right), \hat{\rho}\left(J+\delta J ; l_{c}\right)\right) F_{1}\left(\hat{\rho}\left(J ; l_{c}+\delta l\right), \hat{\rho}\left(J+\delta J ; l_{c}+\delta l\right)\right)},
$$



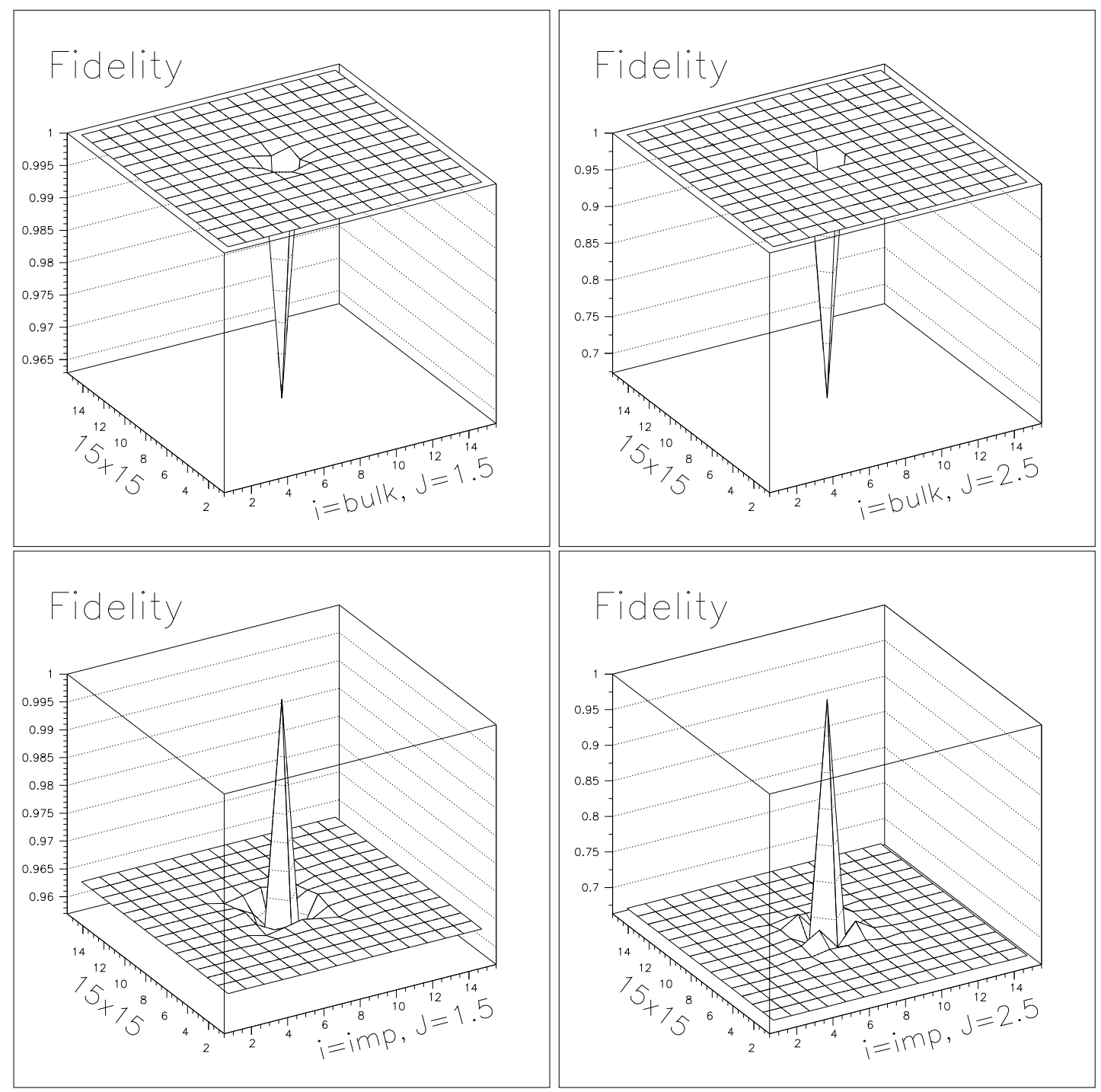

FIG. 3: One-site fidelity, given for fixed values of the parameter $J$, between any site and: a site in the bulk (upper figures); a site in the impurity (lower figures). Left figures are taken for $J^{-}=1.5<J_{0}$, figures on the right for $J^{+}=2.5>J_{0}$, where $J_{0}=1.9$ is the point of the quantum phase transition. Note the drop of the fidelity between the impurity and the bulk state, from $F_{1}^{-} \simeq 0.96$ for $J^{-}=1.5<J_{0}$, to $F_{1}^{+} \simeq 0.65$ for $J^{+}=2.5>J_{0}$ - another signature of the quantum phase transition.

which can be seen as an indicator of total correlations, as in the case of uncorrelated two-site composite systems the above quotient reduces identically to 1 . Therefore, the two-site composite system is necessarily correlated whenever $F_{2} \neq 1$. Note that $C_{2}$ is not the measure of correlations - the two-site composite system can still exhibit nonzero correlations between its one-site subsystems even for $F_{2}=1$. This is clearly seen in Fig. 5 , where $C_{2}=1$ for all values of $J$ except for $J=J_{0}$, although is was shown in [34] that the considered two-site systems are correlated for every value of $J$. Yet, precisely around the point $J_{0}$ of the phase transition, the quantity $C_{2}$ exhibits an abrupt change, thus showing an enhanced change in the amount of the total correlations between the one-site subsystems of an overall two-site system, see Fig. 5. We also see that its numerical value around $J=J_{0}$ approaches 1 as the step $\delta l$ increases - ie the change of total correlations decreases as the distance between the two sites increases. This behavior was already observed before in the study of total and quantum correlations (entanglement) in this model [34]. In connection to this, we note here that the fidelity induces various distance measures on the set of quantum states (for an overview, see for example 22] and references therein), which can further induce fidelity-based multi-particle entanglement measures, based on the quantum state distinguishability, similarly to the case of the well known relative entropy of entanglement measures [36].

In the case of two-site subsystems with both one-site electrons located in the bulk, the fidelity taken between states 


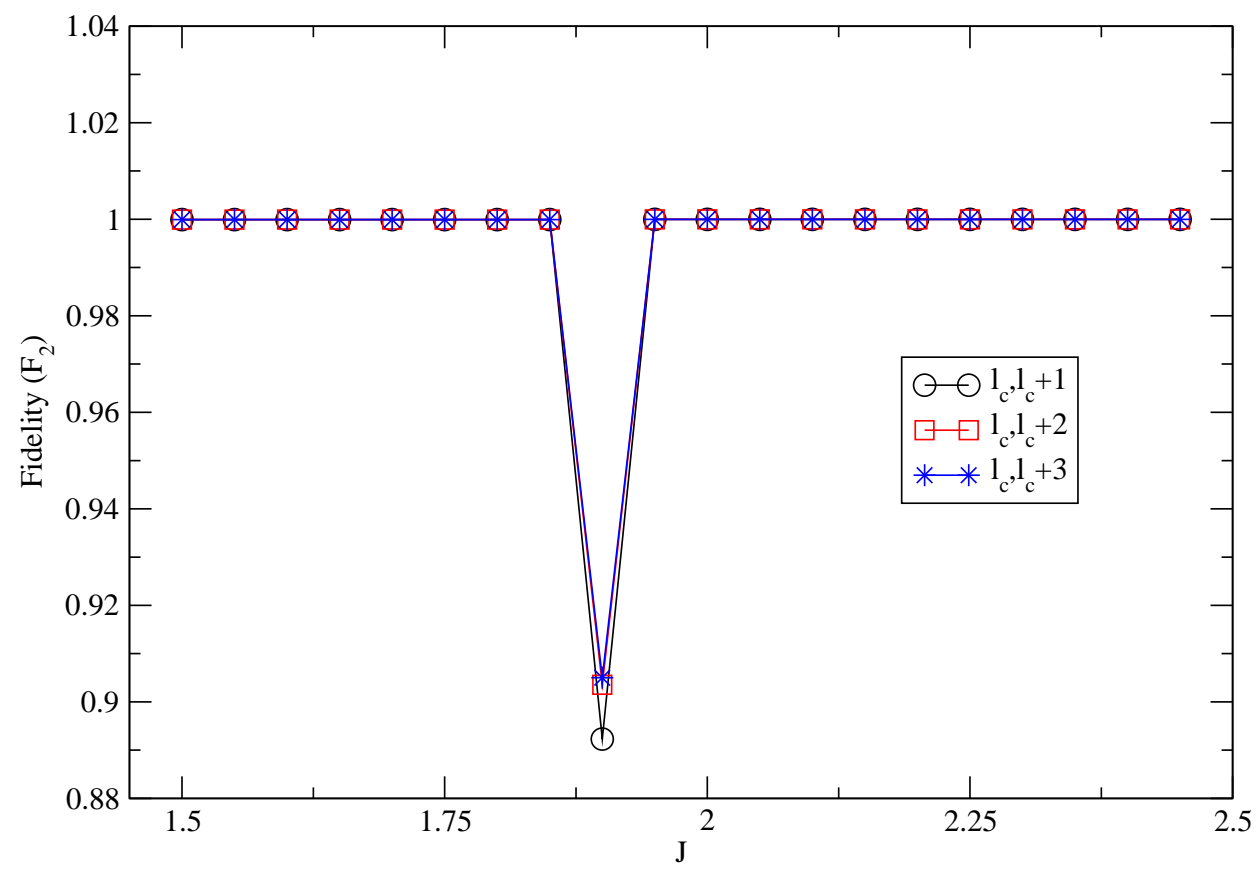

FIG. 4: Two-site fidelity between two states taken for two close parameter points $J$ and $J+\delta J(\delta J=0.05)$, when one site is at the impurity and other in the neighborhood of it. Note the sudden drop of the fidelity around the point of the quantum phase transition, for the case of impurity + neighboring site.

obtained for two close parameter values $J$ and $J+\delta J$, is essentially equal to one (with $\delta J=0.05$ ). Analogously to the case of the fidelity between one-site partial states, here as well we see that the impurity does not affect considerably the partial states of the bulk of the lattice, even around the point of the quantum phase transition. Yet, we have observed a small deviation around $J_{0}$ from the otherwise common value $F_{2} \simeq 1$ which indicates that for larger subsystems (when considering the fidelities $F_{3}, F_{4}$, etc. of the 3-electron and 4-electron subsystems respectively), the impurity might start to increasingly influence the collective behavior of the bulk.

Finally, as in the previous case, we have evaluated the Uhlmann mixed state geometric phase $\phi_{g}$, again obtaining the trivial value $\phi_{g} \simeq 0$ everywhere, apart for the case when one electron site is located at the impurity: the structure of the two-site partial states' eigenvectors is not considerably affected by the quantum phase transition.

\section{CONCLUSION}

In this paper we have applied the concept of fidelity to approach quantum phase transitions, taking as the physical system a conventional 2-dimensional superconductor with a classical magnetic impurity placed at its centre. Unlike previous studies, where the overall states of the system were considered, here we have studied the fidelity for partial one- and two-site states. We have shown that the point of the quantum phase transition is still clearly marked by the sudden drop of the mixed state fidelity, even in the simplest case of the one-site partial states, as long as they are close to the impurity itself. The same holds for the two-site partial states - the fidelity exhibits a sudden drop in the vicinity of the quantum phase transition. Here, for the transition to be inferred it is necessary that one of the subsystem electrons lies at the impurity, or its neighborhood. This is expected to occur for this model since, as shown earlier, the impurity acts mainly as a local perturbation (but see also the behavior of the non vanishing two-site "long distance" entanglement measures discussed in 34]). We have also evaluated the Uhlmann mixed state geometric phase for the cases of both one- and two-site partial states and found it to be trivial (ie equal to one) for every value of the quantum phase transition driving parameter $J$, except when an electron site is at the impurity, thus showing that the structure of the subsystem eigenvectors has little relevance to the magnetic transition discussed. Finally, we have shown that the fidelity approach can also be useful in studying the total correlations between subsystems of the whole physical system, and how the point of quantum phase transition can be inferred from the change in the amount of total correlations (given by the quantity $C_{2}(18)$ ), which is closely related to the fidelity between the partial states discussed in this work. 


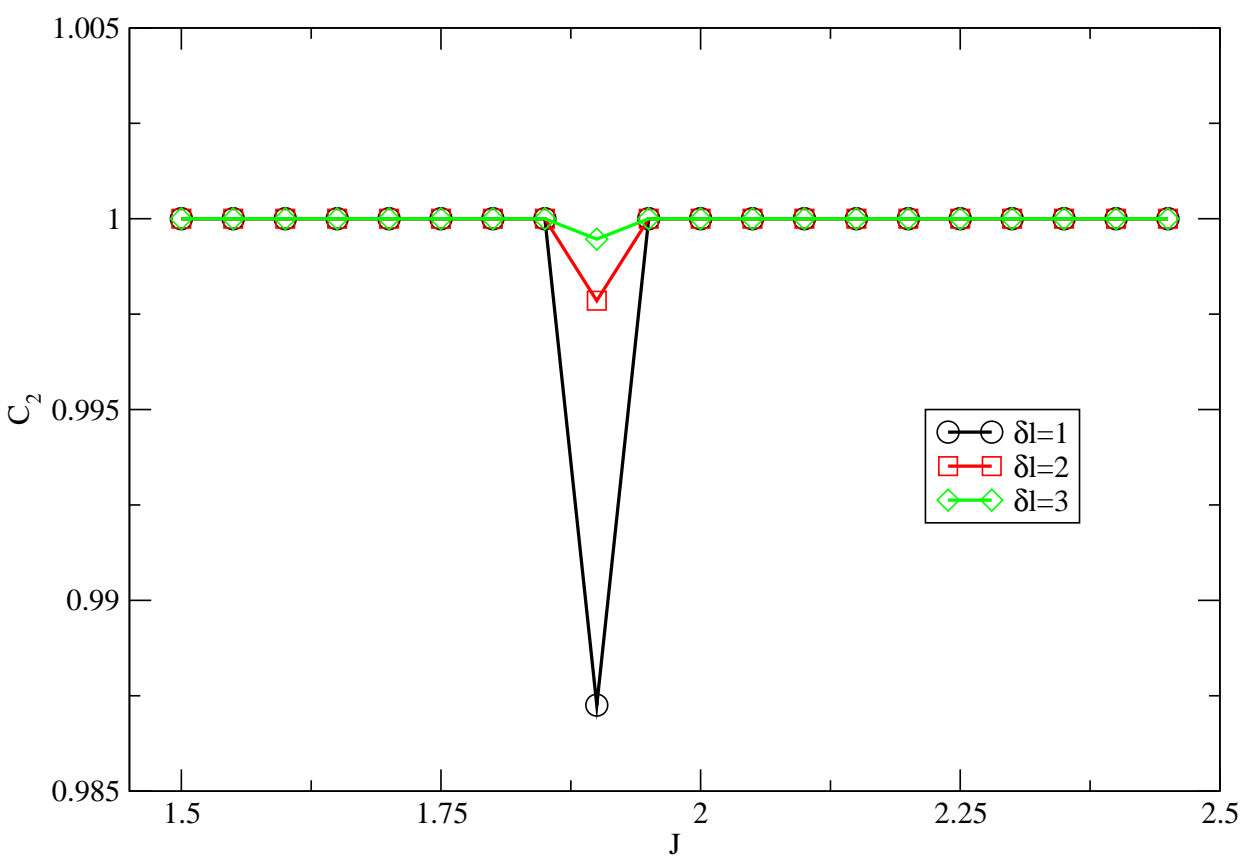

FIG. 5: The $C_{2}$ function between two states taken for two close parameter points $J$ and $J+\delta J(\delta J=0.05)$, when one site is at the impurity and other in the neighborhood of it. Note its sudden drop around the point of the quantum phase transition, as well as the decrease of its deviation from 1 as the other site gets further from the impurity.

We believe that this work offers yet another confirmation of the usefulness of the fidelity approach to study quantum phase transitions. It extends this approach to partial states, showing that, in some cases at least, quantum phase transitions can be signaled out by the behavior of the mixed state fidelity taken between local partial states. It is an interesting and challenging task to try to find a unified view to this new approach and possibly connect it to some previously well studied thermodynamic quantities. Also, the generalization to thermal phase transitions is another possible path of further research. Finally, we see our simplified study of the total correlations in a composite quantum state, based on the fidelity, as just a first step towards exploring the possibility of using the fidelity-based quantum state distinguishability for quantifying correlations, both classical as well as quantum (entanglement), a subject of research that has on its own found rich applications within the field of condensed matter and many-body physics.

\section{ACKNOWLEDGMENTS}

NP thanks the support from Fundação para a Ciência e a Tecnologia (Portugal), grant SFRH/BPD/31807/2006. PDS, PN and VRV acknowledge the support of projects SFA-2-91, the ESF Science Programme INSTANS 2005-2010, POCI/FIS/58746/2004 and FCT POCI/MAT/55796/2004. VKD is supported by the Polish Ministry of Science and Higher Education as research projects in years 2006-2009 and 2007-2010 and by STCU Grant No. 3098 in Ukraine. This work is also supported by the cooperation agreement between Poland and Portugal in the years 2007-2008.

[1] M. A. Nielsen and I. L. Chuang, Quantum Computation and Quantum Information, Cambridge University Press (2000).

[2] S. Sachdev, Quantum Phase Transitions, Cambridge University Press (1999).

[3] Strongly correlated systems, coherence and entanglement, edited by J. M. P. Carmelo, P. D. Sacramento, J. M. B. L. dos Santos and V. R. Vieira, World Scientific, Singapore (2007).

[4] W. K. Wootters, Phys. Rev. D 23, 357 (1981).

[5] P. Zanardi and N. Paunković, Phys. Rev. E 74, 031123 (2006).

[6] P. Zanardi, M. Cozzini and P. Giorda, arXiv:quant-ph/0606130v1; M. Cozzini, P. Giorda and P. Zanardi, arXiv:quant-ph/0608059v1.

[7] P. Buonsante and A. Vezzani, Phys. Rev. Lett. 98, 110601 (2007).

[8] N. Oelkers and J. Links, Phys. Rev. B 75, 115119 (2007). 
[9] S. Chen, L. Wang, S.-J. Gu and Y. Wang, arXiv:0706.0072v1 [cond-mat.other].

[10] M.-F. Yang, arXiv:0707.4574v1 [quant-ph].

[11] H.-Q. Zhou and J. P. Barjaktarevic, arXiv:cond-mat/0701608v1 [cond-mat.stat-mech]; H.-Q. Zhou, J.-H. Zhao and B. Li, arXiv:0704.2940v1 [cond-mat.stat-mech]; H.-Q. Zhou, arXiv:0704.2945 1 [cond-mat.stat-mech].

[12] M. Cozzini, R. Ionicioiu and P. Zanardi, arXiv:cond-mat/0611727v1 [cond-mat.stat-mech].

[13] A. Hamma, W. Zhang, S. Haas and D. A. Lidar, arXiv:0705.0026v1 [quant-ph].

[14] S.-J. Gu, H.-M. Kwok, W.-Q. Ning and H.-Q. Lin, arXiv:0706.2495 2 [quant-ph].

[15] P. Zanardi, P. Giorda and M. Cozzini, arXiv:quant-ph/0701061v1.

[16] L. C. Venuti and P. Zanardi, arXiv:0705.2211v2 [quant-ph].

[17] W.-L. You, Y.-W. Li and S.-J. Gu, arXiv:quant-ph/0701077v2.

[18] P. Zanardi, H. T. Quan, X. Wang and C. P. Sun, Phys. Rev. A 75, 032109 (2007); P. Zanardi, L. C. Venuti and P. Giorda, arXiv:0707.2772 2 [quant-ph].

[19] N. Paunković and V. R. Vieira, arXiv:0707.4667v1 [quant-ph].

[20] A. C. M. Carollo and J. K. Pachos, Phys. Rev. Lett. 95, 157203 (2005); S.-L. Zhu, Phys. Rev. Lett. 96, 077206 (2006); A. Hamma, arXiv:quant-ph/0602091 1; M.E. Reuter, M.J. Hartmann and M.B. Plenio, Proc. Roy. Soc. Lond. A 463, 1271 (2007).

[21] A. Uhlmann, Rep. Math. Phys. 24, 229 (1986).

[22] C. A. Fuchs, Distinguishability and Accessible Information in Quantum Theory, Ph. D. thesis, arXiv: quant-ph/9601020.

[23] A. Sakurai, Prog. Theor. Phys. 44, 1472 (1970).

[24] K. Satori et al., J. Phys. Soc. Jpn. 61, 3239 (1992).

[25] M.I. Salkola, A.V. Balatsky and J.R. Schrieffer, Phys. Rev. B 55, 12648 (1997).

[26] D.K. Morr and N.A. Stavropoulos, Phys. Rev. B 67, R020502 (2003).

[27] D.K. Morr and J. Yoon, Phys. Rev. B 73, 224511 (2006).

[28] A.V. Balatsky, I. Vekhter and J.-X. Zhu, Rev. Mod. Phys. 78, 373 (2006).

[29] A. A. Abrikosov and L. P. Gor'kov, Zh. Eksp. Teor. Fiz. 39, 178 (1960) [Sov. Phys. JETP 12, 1243 (1961)].

[30] R. Ramazashvili and P. Coleman, Phys. Rev. Lett. 79, 3752 (1997).

[31] P.D. Sacramento, V.K. Dugaev and V.R. Vieira, Phys. Rev. B 76, 014512 (2007).

[32] P.G. de Gennes, Superconductivity of Metals and Alloys (Addison-Wesley, Reading, MA, 1989).

[33] J.A.M. Vermaseren, "New features of FORM", arXiv:math-ph/0010025 v2.

[34] P. D. Sacramento, P. Nogueira, V. R. Vieira and V. K. Dugaev, arXiv:0706.1476v1 [cond-mat.supr-con].

[35] J. Tidström and E. Sjöqvist, Phys. Rev. A 67, 032110 (2003).

[36] V. Vedral, M. B. Plenio, M. A. Rippin and P. L. Knight, Phys. Rev. Lett. 78, 2275 (1997).

[37] Note that in the case of pure states, this corresponds to the case of mutually orthogonal state vectors.

[38] These interaction terms are easily expressed in terms of the electron spin operators $\hat{\vec{S}}$ and the Nambu operators $\hat{\vec{T}}$, discussed in detail in [19]. They commute with each other, ie $\left[\hat{T}^{\alpha}, \hat{S}^{\beta}\right]=0$, for every $\alpha, \beta \in\{0,+,-\}$, and they have a common eigenbasis. In fact, these operators satisfy $\hat{T}^{\alpha} \hat{S}^{\beta}=0$, and act non-trivially in mutually orthogonal subspaces, with even and odd number of particle occupation numbers, respectively, annihilating the states in the other subspace. 